Volume 3

\title{
The Relationship of Acculturation and All Cause Mortality Among Mexican Americans: Results from the NHANES III Mortality Follow Up
}

Gloria C. Diaz

Portland State University

Follow this and additional works at: https://pdxscholar.library.pdx.edu/mcnair Let us know how access to this document benefits you.

\section{Recommended Citation}

Diaz, Gloria C. (2009) "The Relationship of Acculturation and All Cause Mortality Among Mexican Americans: Results from the NHANES III Mortality Follow Up," PSU McNair Scholars Online Journal: Vol. 3: Iss. 1, Article 16.

https://doi.org/10.15760/mcnair.2009.106

This open access Article is distributed under the terms of the Creative Commons Attribution-NonCommercialShareAlike 4.0 International License (CC BY-NC-SA 4.0). All documents in PDXScholar should meet accessibility standards. If we can make this document more accessible to you, contact our team. 
Portland State University McNair Research Journal 2009

The Relationship of Acculturation and All Cause Mortality Among Mexican Americans: Results from the NHANES III Mortality Follow Up

by

Gloria C. Diaz

Faculty Mentor:

Carlos J. Crespo

Citation: Diaz, Gloria C. The Relationship of Acculturation and All Cause Mortality Among Mexican Americans: Results from the NHANES III Mortality Follow Up. Portland State University McNair Scholars Online Journal, Vol. 3, 2009: pages [106-117] 


\section{The Relationship of Acculturation and All Cause Mortality Among Mexican Americans: Results from the NHANES III Mortality Follow Up}

GLORIA C. DIAZ

CARLOS J. CRESPO, FACULTY MENTOR

FALLON TAYLOR, MPHC

CHRISTOPHER TORRES, NSCA-CPT, USAW, MCNAIR SCHOLAR

\section{Abstract \\ Background}

Mexican Americans comprise the largest percentage of Hispanics in the United States, accounting for $9.5 \%$ of the population in 2006. Although more than 40\% of Mexican Americans are foreign born, even individuals born in the U.S. face the process of acculturation, or the merging of Mexican and American cultures. Previous research has linked acculturation to both negative and positive health outcomes, with more acculturated Mexican Americans experiencing increased rates of substance use and abuse, asthma, and obesity, but decreased rates of physical inactivity and low self-esteem. The purpose of this study is to examine the relationship between acculturation and all-cause mortality in a nationally representative sample of Mexican Americans.

Methods

Using SAS and SUDAAN, we conducted a survival analysis using data from the Third National Health and Nutrition Examination Survey (NHANES III), a nationwide, multi-stage survey conducted between 1988 and 1994. Our analytic sample consisted of 3,330 Mexican-American adults ages 25-64 years who took part in a home interview and were followed up for mortality until 2002. Language spoken at home was used to assess the level of acculturation with those speaking mostly Spanish at home identified as having lower levels of acculturation.

Results

After adjusting for age, education, presence of chronic diseases and health insurance we found that acculturation was not a predictor of all-cause mortality in this group of Mexican Americans, men or women ages 25-64 years.

Conclusion

These findings indicate that acculturation may have no affect on the mortality of Mexican American men and women younger than 65 years of age. Additional research needs to be conducted in order to further explore other measurements of acculturation and the full spectrum of its effects on morbidity and disease-specific mortality.

\section{Introduction}

The Latino community has increased dramatically in the last ten years and according to recent projections by the United States Census Bureau the population is expected to triple by the year 2050 (1). According to census data from 2006, the largest segment of Latinos within the United States are Mexican Americans (2). Traditionally the Mexican American community has been largely concentrated in states such as California and Texas (1). Compared to the other major Latino groups (Puerto Ricans and Cubans) the 
Mexican American population is relatively young; the median age being 25.7 years old (2). Being that over 40 percent of this population is foreign born (2) one can assume that many members of the Mexican American community will experience acculturation to some degree within their lifetimes. It would be remiss to not acknowledge acculturation when looking at the Mexican American population, particularly when it comes to health status.

Acculturation is defined as "the process by which groups or individuals integrate the social and cultural values, ideas, beliefs, and behavioral patterns of their culture of origin with those of a different culture" (3). The process of acculturation should not be seen as one single event in a person's life but rather a long and continuous process that can positively or negatively affect the health of recent immigrants and their offspring (4, $5,6)$. The process of acculturation is a multi-dimensional, long term process that has varying degrees of effect on individuals not only between different ethnic groups but within them as well (7). Therefore, research exploring the association between acculturation and health status should be continued.

While the Latino population may suffer from health disparities and low socioeconomic status, the mortality rates are similar to those of non-Hispanic Whites and much lower than non-Hispanic Blacks. This contrast is commonly referred to as the Hispanic/Latino Paradox. Extensive research has been carried out aiming to identify the factors that contribute to this paradox and what these "protective" factors may be. Current research has linked greater acculturation with obesity, smoking, erosion of diet quality, high alcohol intake, and with less physical inactivity among Mexican Americans (8, 9, 10,11). There continues to be mixed results on the benefits of acculturation; therefore it is inherent to continue research regarding its place within the context of the Mexican American experience, as well as within other Latino groups. Obtaining this information will further contribute to our knowledge on the unique effects migration has on the Mexican American population and may assist public health professionals design more specific health programs for these communities. 
In this study we examine the relationship between acculturation and all cause mortality within the Mexican American community using data from the Third National Health and Nutrition Examination Survey (NHANES III). In addition, we hope to identify acculturation constructs that may modify social determinants of health disparities in a large sample of Mexican Americans.

\section{Methods}

Data for this study was drawn from NHANES III, conducted between 1988 and 1994 by the Centers for Disease Control and Prevention's National Center for Health Statistics. National data was collected on the health and nutritional status of 33,994 citizens aged two months and older, excluding those residing in institutionalized settings (military personal and prisons), through in person interviews and physical examinations. NHANES III oversampled the two largest racial/ethnic minority groups in the US, Mexican Americans and African Americans, in order to generate statistically reliable results during analysis. Weights were assigned to each participant allowing their responses to represent the racial/ethnic group they identify within the US. Participants were interviewed in their homes by trained bilingual (Spanish/English) interviewers. Our final sample size consisted of 3,330 self identified Mexican Americans between 25 and 64 years of age.

\section{Measures}

Through the NHANES III at home questionnaire, participants were asked what their primary language spoken at home was. Language mainly spoken at home (Spanish, Spanish/English, and English) was used as the measurement of acculturation. Those who spoke mainly Spanish at home were defined as being the least acculturated and those who spoke mainly English at home were defined as being the most acculturated.

Information on all-cause mortality, our dependent variable, was obtained through the National Death Index (NDI), a repository of all death certificates in the United States, Puerto Rico and all US territories. NHANES III records were linked to the NDI in 2000 creating the NHANES III linked mortality file allowing the study of associations between health and risk factors with mortality. Other variables of interest included were: age, education, presence of chronic disease and health insurance. Wanting to focus on those who would have been 
less likely to have insurance and on the young immigrant population our participants were between the ages of 25-64 (12). Education was measured as: $<8$ years, 9-11 years, 12years, 13 years $\geq$. Participants were identified with having chronic disease if they responded "yes" to having any of the following diseases: arthritis, bronchitis, stroke, asthma, congestive heart failure, emphysema, heart attack, osteoporosis, cancer, or back pain. To assess if participants had health insurance they were questioned if they had been covered by any type of health insurance in the past month. Acceptable forms of health insurance were Medicare, Medicaid, military health care, privately obtained insurance, or insurance through a current or former employer or union.

\section{Data Analysis}

SUDAAN (Research Triangle Institute, Research Triangle Park, NC) was used in order to calculate the frequency and percentage of the participants' demographic information. Due to the complex nature of the NHANES III sampling design, SUDAAN incorporated sampling weights that adjusted for the unequal sample size in the Mexican American population. Further frequency distribution data was analyzed for those "Assumed Alive" and "Assumed Deceased". We also used SAS, Version 9.1 (SAS Institute Inc, Cary, NC), to estimate the hazard ratio for acculturation and all cause mortality in men and women respectively. Sampling weights were also used to adjust for small sample size and discrepancies in selection probabilities. 


\section{Results}

The descriptive analysis presented in Table 1, describes the participants' demographics by age, education, income, work status, place of birth, years in the US, and preferred language at home. Our cohort of Mexican American men and women was nearly equally divided at $50.1 \%$ and $49.8 \%$ respectively. A majority $(65 \%)$ of our cohort had less than 12 years of education. Of our participants $23.7 \%$ of the men and $22.09 \%$ of the women were born in Mexico and 26.21\% of men and 27.98\% of women were born in the United States. For purposes of this study results were adjusted by age, education (<8y, 9-11 y, $12 \mathrm{y}, 13>$ ), insurance (yes, no), and the presence of chronic disease (yes, no).

Measurement of acculturation was based on language preference at home. Approximately 59\% of our participants preferred to speak Spanish at home, with approximately 37\% of our participants preferring to speak English at home and 3.81\% of them spoke both at home. Participants who spoke both Spanish and English at home were not included in the study due to insignificant sample size. At the time the NHANES III linked mortality file was created, 66 of female participants and 94 male participants were assumed dead. 
Table 1-Descriptive Demographics

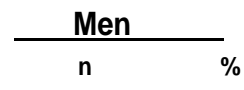

Women

n

$\begin{array}{lr}621 & 18.65 \\ 501 & 15.05 \\ 269 & 8.08\end{array}$

1229

918

36.93

27.58

14.39

55-64 y

$304 \quad 9.13$

\section{Education y}

$\begin{array}{lll}\leq 8 \mathrm{y} & 761 & 23.1 \\ \mathbf{9}-11 \mathrm{y} & 251 & 7.6 \\ 12 \mathrm{y} & 325 & 9.8 \\ 13 \mathrm{y} \geq & 311 & 9.4\end{array}$

$\begin{array}{lr}707 & 21.47 \\ 277 & 8.41 \\ 387 & 11.75 \\ 274 & 8.32\end{array}$

Income

$\begin{array}{lll}<\mathbf{2 0 , 0 0 0} & 706 & 21.97 \\ \mathbf{2 0 - 3 4 , 9 9 9} & 394 & 12.26 \\ >\mathbf{3 5 , 0 0 0} & 507 & 15.78\end{array}$

Work Status

\section{Employed}

$1358 \quad 41.4$

Unemployed

$118 \quad 3.6$

Not in Labor

$172 \quad 5.24$

Force

Place of Birth

$\begin{array}{lll}\text { Mexico } & 756 & 24.48\end{array}$

United States

$\begin{array}{ll}756 & 24.48 \\ 779 & 25.23\end{array}$

$705 \quad 22.83$

$848 \quad 27.46$

\section{Years in U.S. $y$}

$<5$ y

5-19 y

10-19 y

$20+y$

Born in U.S.

$\begin{array}{lr}205 & 6.64 \\ 115 & 3.72 \\ 275 & 8.91 \\ 161 & 5.21 \\ 779 & 25.23\end{array}$

\section{Language}

Eng at Home

Span at Hom

Eng/Span at

$589 \quad 17.83$

$1010 \quad 30.58$

$58 \quad 1.76$

Eng at Work

Span at Worl

Eng/Span at '

$\begin{array}{lr}771 & 33.83 \\ 486 & 21.33 \\ 115 & 5.05\end{array}$

$\begin{array}{lr}229 & 7.42 \\ 101 & 3.27 \\ 234 & 7.58 \\ 141 & 4.57 \\ 848 & 27.46\end{array}$

$626 \quad 18.95$

$952 \quad 28.82$

$68 \quad 2.06$

$568 \quad 24.92$

$276 \quad 12.11$

$63 \quad 2.76$
1375

1521

44.56

49.29

5.37

23.73

22.3

29.8

$\begin{array}{rl}105 & 3.27 \\ 34 & 1.06 \\ 49 & 1.53\end{array}$

Assumed Deceased

n

$\%$

1.06

$\begin{array}{rrrr}2131 & 65.01 & 92 & 2.81 \\ 176 & 5.37 & 15 & 0.46 \\ 778 & 23.73 & 86 & 2.62\end{array}$

2.62

$\begin{array}{rrrr}396 & 12.83 & 38 & 1.23 \\ 211 & 6.84 & 5 & 0.16 \\ 487 & 15.78 & 22 & 0.71 \\ 281 & 9.11 & 21 & 0.68 \\ 1521 & 49.29 & 104 & 3.37\end{array}$

$\begin{array}{rr}1159 & 35.11 \\ 1829 & 55.41 \\ 119 & 3.6 \\ & \\ 1292 & 56.74 \\ 718 & 31.53 \\ 172 & 7.55\end{array}$

$\begin{array}{rl}54 & 1.64 \\ 133 & 4.03 \\ 7 & 0.21 \\ & \\ 46 & 2.02 \\ 44 & 1.93 \\ 5 & 0.22\end{array}$




\section{Table 2}

Acculturation and All Cause Mortality in Mexican American Men and Women aged 25-64 years (Main language at home).

\begin{tabular}{lll} 
Women & HR & $\mathbf{9 5 \% ~ C I ~}$ \\
\hline English & 1 & (Reference) \\
Spanish & 1.37 & $0.69-2.72$ \\
& & \\
Men & HR & $\mathbf{9 5 \% ~ C I ~}$ \\
\hline English & 1 & (Reference) \\
Spanish & 0.81 & $0.46-1.45$
\end{tabular}

Note: $\mathrm{HR}=$ Hazards Ratio. $\mathrm{CI}=$ Confidence Interval. HRs are adjusted for age $(\leq 8 \mathrm{y}, 9-$ $11 \mathrm{y}, 12 \mathrm{y}, 13 \geq$ ) gender (male, female), health insurance (insured, uninsured), and presence of chronic disease (yes/no).
Table 2 describes the adjusted hazard ratios and $95 \%$

confidence intervals (CIs) for acculturation and all cause

mortality in Mexican American men and women separately.

There was no statistically significant association between

acculturation (measured by language preference in the home) and all cause mortality for both men $(p>0.47)$ and women $(p>0.37)$. However, acculturation and all cause mortality was statistically significant in men who were uninsured ( $p>0.02)$. Our study shows that they were two times more likely (HR 2.04, 95\% CI 1.15-3.61) to die than men who were insured. This finding is congruent with past research done on the uninsured and all cause mortality.

Another interesting finding was that the direction of the hazard ratio calculated for men (HR 0.81, 95\% CI $0.45-1.45$ ) and women (HR 1.37, 95\% CI 0.69-2.72) was incongruent. Meaning that, all cause mortality is less likely to occur in men who are less acculturated than men who are more acculturated.

\section{Discussion}

Our results indicate that there is no relationship between acculturation and all cause mortality. The strengths of our study lie in the well characterized standard methods used in collecting data for NHANES III as a nationally representative sample. The questionnaire used to collect the information used in this study is validated and was conducted by well trained bi-lingual interviewers. Yet, limitations exist in our acculturation measurement in that one variable such as language spoken at home is limited and is not capable of capturing the acculturation experience. Further research using NHANES III data needs to be conducted exploring other acculturation measures for this population. 
The process of acculturation is complex and multifaceted, factors that are enhanced based on country of origin, gender, or out of home interactions (13). The process can also be further complicated based on factors such as family structure, religious beliefs, skin color, and attitudes of the majority culture (7). It is important to consider these factors when examining the health status of communities comprised of immigrants and of those whose ethnic distinctiveness may make it difficult for them to assimilate or "fit into" the dominate culture. Other factors such as age at migration and social interaction can also affect health (5). Although individuals may have been born in the United States and speak primarily English they may exist in a bi-cultural world in which they balance between Anglo and Latino identity. For example, in a recent study on immigrant status and acculturation participants identified more closely as being Latino, however the mean score was closer to "bicultural" based on Marín’s Acculturation Scale (14). Some researchers argue that acculturation should not be studied as a linear process but rather as a multicultural identity existing between cultures and that more emphasis should be placed on cultural strengths and structural risks factors $(15,17)$.

Within our study we found no significant association between acculturation (measured by language spoken at home) and all cause mortality. Research regarding acculturation and health continues to produce mixed and contrasting results. For example, while some studies have linked more highly acculturated Mexican Americans with greater obesity or erosion of diet quality $(9,10)$ others found that more highly acculturated Mexican Americans are more physically active during leisure time (8). In a recent study a majority of participants had reported that their health and overall quality of life had improved since living in the United States despite that a majority of them had also reported gaining weight (18). In regards specifically to Latinas, those who are highly acculturated have been found to have higher BMIs, smoke more and consume more alcohol than their less acculturated counter parts (11). However, they are more likely to exercise and to take preventative measures such as receiving breast cancer screenings $(11,19)$. While research regarding acculturation has been done to further fully understand migration and its effects on immigrant health, it has also been conducted in an attempt to identify reasons for the existence of the Hispanic/Latino Paradox. 
The idea of the "Hispanic Paradox" was originally presented in "The Health of Hispanics in the Southwestern United States: an Epidemiologic Paradox” written by Markides and Coreil in 1986 (20,21). Over the last two decades much controversy has surrounded the Latino/Hispanic Paradox, framed by three primary explanation theories for the paradox's existence $(20,22,23,24,25)$. The "healthy migrant" hypothesis supposes that migrants to the United States are the healthiest members of the communities from which they come from (11). The "salmon bias" theory, suggests that mortality statistics may be skewed by Latinos returning to their countries of origin at the end of their lives to eventually die there (11). If this is the case some feel that it would create inaccuracies when calculating mortality rates however, others feel that the "salmon bias" isn't relevant for all Latino populations (22). Finally, some researchers have argued that the Latino paradox resulted from miscalculations of mortality rates for Latinos in the United States due to incorrect identification in regards to ethnicity and race on death certificates and to inaccurate numbers for population and deaths among Latinos $(26,27)$.

In our study there was a significant link between acculturation and all cause mortality amongst MexicanAmerican men who were uninsured. Reports have shown that Latinos in the United States are less likely to possess health insurance than non-Hispanic whites, non-Hispanic blacks or non-Hispanic Asians. (28, 29, 30) A recent report on health insurance coverage by the National Health Interview Survey found that of the Latinos surveyed $1 / 3$ were uninsured at the time of the interview or had been uninsured for at least part of the past year, and had been without insurance over a year (29). One study found that in 1999 for those who were uninsured almost half had not received needed care (i.e. doctor visit, prescribed medication, or treatments) when needed (31). While research has been done identifying cultural barriers as an issue for Latino health care other barriers appear to be more relevant to the population $(32,33,34,35)$. Estrada et al. found, using NHANES data that of their participants 33\% reported encountering one or more barrier to health care and of those $73 \%$ reported that the barriers were enough for them to prevent from obtaining health care on that occasion (35). However as opposed to cultural barriers, for their participants their top five encountered barriers were costs, child care, transportation, and losing pay from work (35). 
As the Latino population continues to grow surely research in this community will continue to increase. When exploring something as complex as acculturation our goal is not so much to identify a "protective factor" that has Latinos living longer but rather to recognize that the Latino community is not homogenous, not only in regards to ethnic variations but also in regards to immigration status and transitional process into life in the United States $(27,36)$. As well as to recognize that health in Latino communities (and all communities) should be, according to Markides, "more importantly tied to improving quality of life in these communities through education, employment, and access to care" (27). By exploring various factors existing within the Latino community researchers should continue to attempt to identify the strengths of individual ethnic communities. By doing so we will be able to build upon these strengths creating more relevant and appropriate disease prevention programs, in turn building healthier and stronger communities.

\section{References}

1. Bernstein R, Edwards T, United States. Bureau of the Census. National population projections. [Washington, D.C.]: U.S. Census Bureau, 2008.

2. United States. Bureau of the Census. American factFinder. Washington U.S. Census Bureau, 1991.

3. VandenBos GR, American Psychological Association. APA dictionary of psychology. Washington, DC: American Psychological Association, 2007.

4. Abraido-Lanza AF, Armbrister AN, Florez KR, et al. Toward a theory-driven model of acculturation in public health research. Am J Public Health 2006;96:1342-6.

5. Angel JL, Angel RJ. Age at migration, social connections, and well-being among elderly Hispanics. J Aging Health 1992;4:480-99.

6. Marín G, Marín BV. Research with Hispanic populations. Newbury Park: Sage Publications, 1991.

7. Padilla AM, Perez W. Acculturation, social identity, and social cognition: A new perspective. Hisp J Behav Sci 2003;25:35-55.

8. Crespo CJ, Smit E, Carter-Pokras O, et al. Acculturation and leisure-time physical inactivity in Mexican American adults: results from NHANES III, 1988-1994. Am J Public Health 2001;91:1254-7.

9. Gordon-Larsen P, Harris KM, Ward DS, et al. Acculturation and overweight-related behaviors among Hispanic immigrants to the US: the National Longitudinal Study of Adolescent Health. Soc Sci Med 2003;57:2023-34.

10. Kaplan MS, Huguet N, Newsom JT, et al. The association between length of residence and obesity among Hispanic immigrants. Am J Prev Med 2004;27:323-6. 
11. Abraido-Lanza AF, Chao MT, Florez KR. Do healthy behaviors decline with greater acculturation? Implications for the Latino mortality paradox. Soc Sci Med 2005;61:1243-55.

12. Singh GK, Siahpush M. Ethnic-immigrant differentials in health behaviors, morbidity, and cause-specific mortality in the United States: an analysis of two national data bases. Hum Biol 2002;74:83-109.

13. Arcia E, Skinner M, Bailey D, et al. Models of acculturation and health behaviors among Latino immigrants to the US. Soc Sci Med 2001;53:41-53.

14. Sussner KM, Lindsay AC, Greaney ML, et al. The Influence of Immigrant Status and Acculturation on the Development of Overweight in Latino Families: A Qualitative Study. J Immigr Minor Health 2008.

15. Phinney J. Ethnic Identity and Acculturation. In: Chun KM, Balls Organista P, Marín G, eds. Acculturation: Advances In Theory, Measurement, and Applied Research. Washington D.C.: American Psychological Association, 2003:63-82.

16. Finch BK, Vega WA. Acculturation stress, social support, and self-rated health among Latinos in California. J Immigr Health 2003;5:109-17.

17. Carter-Pokras O, Zambrana RE. Latino Health Status. In: Aguirre-Molina M, Molina C, Zambrana RE, eds. Health Issues in the Latino Community. San Francisco: Josey-Bass, 2001:23-54.

18. Kepka D, Ayala GX, Cherrington A. Do Latino immigrants link self-rated health with BMI and health behaviors? Am J Health Behav 2007;31:535-44.

19. O'Malley AS, Kerner J, Johnson AE, et al. Acculturation and breast cancer screening among Hispanic women in New York City. Am J Public Health 1999;89:219-27.

20. Markides KS, Eschbach K. Aging, migration, and mortality: current status of research on the Hispanic paradox. J Gerontol B Psychol Sci Soc Sci 2005;60 Spec No 2:68-75.

21. Markides KS, Coreil J. The health of Hispanics in the southwestern United States: an epidemiologic paradox. Public Health Rep 1986;101:253-65.

22. Abraido-Lanza AF, Dohrenwend BP, Ng-Mak DS, et al. The Latino mortality paradox: a test of the "salmon bias" and healthy migrant hypotheses. Am J Public Health 1999;89:1543-8.

23. Turra CM, Goldman N. Socioeconomic differences in mortality among U.S. adults: insights into the Hispanic paradox. J Gerontol B Psychol Sci Soc Sci 2007;62:S184-92.

24. Smith DP, Bradshaw BS. Rethinking the Hispanic paradox: death rates and life expectancy for US non-Hispanic White and Hispanic populations. Am J Public Health 2006;96:1686-92.

25. Hunt KJ, Resendez RG, Williams K, et al. All-cause and cardiovascular mortality among Mexican-American and non-Hispanic white older participants in the San Antonio Heart Study - Evidence against the "Hispanic paradox". American Journal of Epidemiology 2003;158:1048-57.

26. Palloni A, Arias E. Paradox lost: explaining the Hispanic adult mortality advantage. Demography 2004;41:385-415.

27. Vega WA, Amaro H. Latino outlook: good health, uncertain prognosis. Annu Rev Public Health 1994;15:39-67.

28. Balluz L, Okoro C, Strine T. Access to Health-Care and Preventative Services Among Hispanics and Non-Hispanics---United States, 2001--2002. Morbidity and Mortality Weekly Report 2004;53:937-41.

29. Cohen RA, Martinez ME, Free HL. Health Insurance Coverage: Early Release of Estimates from the National Health Interview Survey, January - September 2007. (8/08/2008, 2008). (http://www.cdc.gov/nchs/data/nhis/earlyrelease/insur200806.htm).

30. Documet PI, Sharma RK. Latinos' health care access: financial and cultural barriers. J Immigr Health 2004;6:5-13. 
31. Carillo JE, Trevino F, Betancourt JR, et al. Latino Access to Health Care. In: Aguirre-Molina M, Molina C, Zambrana RE, eds. Health in the Latino Community. San Francisco: JoseyBass, 2001:55-73.

32. Chesney AP, Chavira JA, Hall RP, et al. Barriers to medical care of Mexican-Americans: the role of social class, acculturation, and social isolation. Med Care 1982;20:883-91.

33. Berk ML, Schur CL, Chavez LR, et al. Health care use among undocumented Latino immigrants. Health Aff (Millwood) 2000;19:51-64.

34. Marshall KJ, Urrutia-Rojas X, Mas FS, et al. Health status and access to health care of documented and undocumented immigrant Latino women. Health Care Women Int 2005;26:916-36.

35. Estrada AL, Trevino FM, Ray LA. Health care utilization barriers among Mexican Americans: evidence from HHANES 1982-84. Am J Public Health 1990;80 Suppl:27-31.

36. Bagley SP, Angel R, Dilworth-Anderson P, et al. Adaptive health behaviors among ethnic minorities. Health Psychol 1995;14:632-40. 